simplistic mathematical generalizations.

Both books provide hours of truly pleasurable reading and also clear and deep insights into the fascinating biology of islands, the struggles to construct comprehensible theories for difficult processes and the techniques for preserving species diversity. They also both introduce a fascinating cast of investigators and entrepreneurs in these fields and islands. Quammen's work, however, must be read with a grain of salt because of his failure to understand the theory and his uncomfortable hagiography of some of the scientists he interviewed.

Lawrence B. Slobodkin is in the Department of Ecology and Evolution, State University of New York at Stony Brook, Stony Brook, New York 11794, USA.

\section{Balancing act}

\section{Miles Padgett}

Lasers and Electro-Optics: Fundamentals and Engineering. By Christopher C. Davis. Cambridge University Press: 1996. Pp. 720. £80, \$125 (hbk); £29.95, $\$ 44.95$ (pbk).

HARDLY a day passes without yet another book appearing on lasers and optoelectronics. Budding authors must strike a balance between heavyweight optical physics and modern device technology. Too much technology and the book is out of date before it reaches the library shelves; too little and it ceases to be relevant to this scientifically fertile and commercially expanding field.

Pitched at specialist undergraduate or graduate students, this book can be warmly recommended. It covers most aspects of laser physics, from basic rate equations to resonator design, is liberally supported by examples of the latest technology and contains a healthy selection of references. There are strong sections on optical systems, including ray propagation, Gaussian beams, fibres and detectors. Towards the end, especially for readers who have found the earlier parts all too easy, there is an excellent chapter on coherence theory.

In a general text such as this, much must be left out. That explains why there is no mention of SEED devices in the parts on technology, or of Stokes parameters in the section on the optical systems, or of optical data storage in the coverage of applications. But these omissions do not detract from the achievement and value of the volume as a whole.

Miles Padgett is at the School of Physics and Astronomy, University of St Andrews, St Andrews, Fife KY16 9SS, UK.

\section{Easy learning}

\section{Patrick Naylor}

Pattern Recognition and Neural Networks. By B. D. Ripley. MIT Press: 1996. Pp. 403. £29.95, \$49.95.

KNOWN for his hype-free approach to neural networks, Brian Ripley here provides an excellent text on the statistics of pattern classifiers and the application of neural network techniques. With an emphasis on the statistical approach, it would have been all too easy for a book of this type to become hard work to read. Ripley has managed, however, to produce an altogether accessible text, aided by examples using both synthetic and real-world data sets. These include case studies on, believe it or not, Leptograpsus crabs and diabetes in Pima Indians.

The scope of the book is wide, and incorporates neural networks initially as methods for learning the parameters of multi-dimensional input-output relationships; only later on does Ripley make passing reference to any architectural equivalence to the brain. The opening chapter on basic decision theory and Bayes rule is substantial and leads to a development of parametric models. Other chapters cover a range of topics such as linear discriminant analysis, multilayer perceptrons and radial basis function networks, as well as unsupervised methods.

The inevitable comparison between this book and C. M. Bishop's Neural Networks for Pattern Recognition (Oxford University Press, 1995) suggests that while Bishop's book is earning a reputation for depth and rigour, Ripley's text will be rightly popular with newcomers to the area for its ability to present the mathematics of statistical pattern recognition and neural networks in an accessible format and engaging style.

Patrick Naylor is in the Department of Electrical and Electronic Engineering, Imperial College, London SW7 2BT, UK.

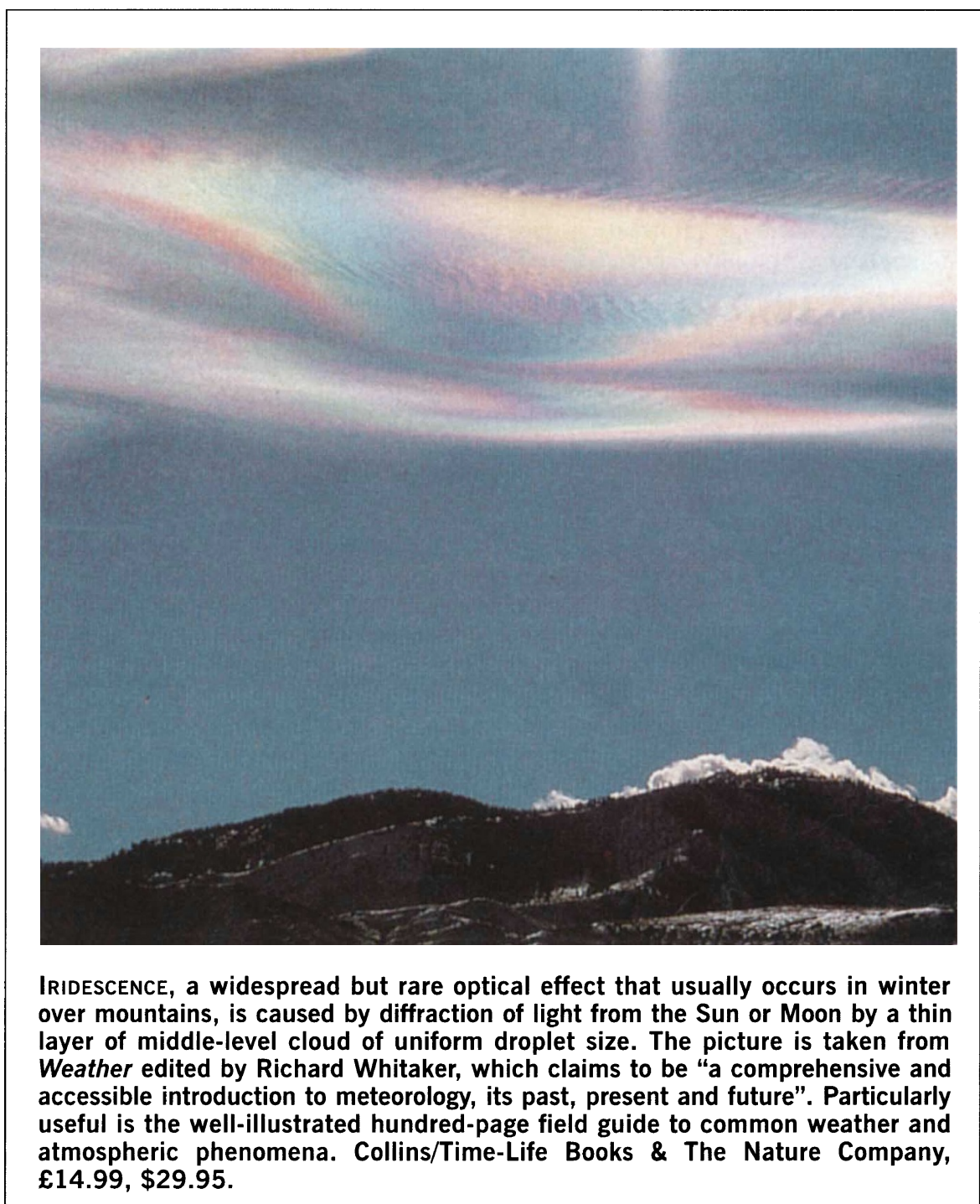

